\title{
Clinical Decision-Making: Developing a 4 C Model Using Graph Theoretic Approach
}

\author{
Rajesh P. Mishra ${ }^{1}$, Nidhi Mundra ${ }^{1}$, Girish Upreti ${ }^{2}$, Marcela Villa-Marulanda ${ }^{3}$ \\ ${ }^{1}$ Mechanical Engineering Department, Birla Institute of Technology and Science Pilani, Pilani, India \\ ${ }^{2}$ Department of Engineering, Methodist University, Fayetteville, NC, USA \\ ${ }^{3}$ Industrial Engineering, Universidad Pontificia Bolivariana, Bucaramanga, Colombia \\ Correspondence: Girish Upreti, ${ }^{2}$ Department of Engineering, Methodist University, Fayetteville, NC, USA.
}

Received: Mar. 22, 2020 Accepted: May 29, 2020 Online Published: June 10, 2020

doi:10.11114/set.v7i1.4781 URL: https://doi.org/10.11114/set.v7i1.4781

\begin{abstract}
The purpose of this paper is to propose a graph-theoretic mathematical model to measure how conducive the environment of a hospital is for decision-making. We propose a 4-C model, developed from four interacting factors: confidence, complexity, capability, and customer. In this graph-theoretic model, abstract information regarding the system is represented by the directed edges of a graph (or digraph), which together depict how one factor affects another. The digraph yields a matrix model useful for computer processing. The net effect of different factors and their interdependencies on the hospital's decision-making environment is quantified and a single numerical index is generated. This paper categorizes all the major factors that influence clinical decision-making and attempts to provide a tool to study and measure their interactions with each other. Each factor and each interaction among factors are to be quantified by healthcare experts according to their best judgment of the magnitude of its effect in a local hospital environment.

A hospital case study is used to demonstrate how the 4-C model works. The graph-theoretic approach allows for the inclusion of new factors and generation of alternative environments by a combination of both qualitative and quantitative modeling. The 4-C model can be used to create both a database and a simple numerical scale that help a hospital set customized guidelines, ranging from patient admittance procedures to diagnostic and treatment processes, according to its specific situation. Implementing this methodology systematically can allow a hospital to identify factors that will lead to improved decision-making as well as identifying operational factors that present roadblocks.
\end{abstract}

Keywords: clinical decision making, graph theory, confidence, complexity, capability, and customer

\section{Introduction}

Broadly, health care can be defined as the diagnosis, treatment, and prevention of illness, disease, injuries, and impairments in people. A hospital has a complex and dynamic environment that requires coordination and communication among various departments. Every case requires different decisions to be made about different treatments but uses the same set of resources. Such decisions range from how elective or emergency surgeries are to be scheduled (Institute of Medicine Committee on the Future of Emergency Care in the US Health System 2006) to which tests and treatments are to be prescribed to a patient, to the integration and coordination of the various hospital services (McGinnis et al., 2013).

These decisions are driven by four main factors: medical professionals' confidence in their acceptance to treat the patient; complexities in the handling and movements of the patient from the time of admission to the time of discharge through the various departments of the hospital; the management's capability to direct information, resources, and actions; and the patient's or customer's perception of the hospital's ability to provide adequate care using its resources.

Each of the four factors is defined by different variables. Confidence, for example, is determined by the condition of the patient, the process of treatment, the uncertainty of action and the uncertainty of outcome. Complexity is determined by planning, integration, coordination, and collaboration in the hospital, and in turn the level of complexity determines how smoothly the flow of personnel and material takes place within a hospital. Capability is determined by the hospital's resource management, service quality, demand variability, demand uncertainty, personnel skill sets, hospital culture, and sustainability, and those factors in turn affect the outcome of a patient's life. 
This paper separates these factors into categories that influence clinical decision-making in order to provide a tool to study and calculate each factor's interactions with others. The objective of this 4-C model exercise is to find a way to measure how conducive the environment of a hospital is to decision-making. Thus, the paper demonstrates how the working environment of a hospital can be quantified for the purpose of better clinical decision-making. We first introduce decision-making tools presently used in the hospital industry and discuss the different factors that affect the conduciveness of hospital environments to clinical decision-making. Lastly, we explain the graph-theoretic approach and demonstrate its use by means of a case study using an Indian hospital.

Table 1. List of abbreviations

\begin{tabular}{lcl}
\hline S. No. & Abbreviation & Full Term \\
\hline 1. & TQM & Total Quality Management \\
2 & FMEA & Failure Mode and Effects Analysis \\
3. & AHP & Analytic Hierarchy Process \\
4. & VPM & Variable Permanent Matrix \\
5. & VPF & Variable permanent function
\end{tabular}

Above are the abbreviations used in this paper.

\section{Literature Review}

Stresses on hospitals such as the COVID-19 pandemic demonstrate the urgency of creating frameworks that can help healthcare professionals and policy-makers make quick, correct decisions both in emergencies and in normal times. Many researchers have attempted to create hospital decision-support models to organize healthcare data for easy retrieval (Trivedi \& Daly (2007); Chang, Hwang, Hung, \& Li (2007); Wright \& Sittig (2008); Santelices et al. (2010); Xu \& Shen (2013); Al-Kadi (2015); Zhang, Tian, Zhou, Araki, \& Li (2016); Piri, Delen, Liu, \& Zolbanin (2017); Abdel-Basset, Manogaran, Gamal, \& Chang (2019)). With the help of such classification systems, patients with varied conditions can be more effectively categorized and appropriate medical care can be provided to them.

The hospital delay is not inevitable and that there is a need to recognize it as essentially a flow issue (Haraden \& Resar, 2004). The emergency department, intensive care unit, operating rooms, and their pre- and post-care areas tend to be viewed as interdependent separate systems rather than a single system. Coordination between the different departments and facilities is required to ensure the smooth flow of patients in the hospital. However, these areas are major bottlenecks both because they are non-interchangeable resources and because they are the primary gatekeepers of effective care.

In 1996, Berwick propounded a "central law of improvement": "Every system is perfectly designed to achieve the results it achieves." A system can be a doctor's practice, a hospital, or a national healthcare system. He focused on redesigning systems to achieve specific and improved results in the context of healthcare delivery, introducing Nolan's model (Angley, Nolan \& Nolan 1992), which begins by setting specific and measurable goals. He also applied the concepts of value and continuous flow, cornerstones of lean manufacturing, to healthcare provision. Chassin (1998), Ferlie and Shortell (2001), and Counte and Meurer (2001) have mentioned about the importance of quality improvement in health care. Kollberg, Dahlgaard, \& Brehmer (2007) have focused on the patient as the primary customer of healthcare services since the patient justifies the existence of such services.

The traditional paternalistic model of medical decision-making, in which doctors make decisions on behalf of their patients, has become outdated (Stevenson, 2000). Instead, Veatch (1972), Charles, Gafni, and Whelan, (1997), Elwyn, Edwards, and Kinnersley (1999), Elwyn, Edwards, Kinnersley \& Grol (2000) have all advocated a shared decisionmaking model. Safford and Allison (2007), more specifically, have proposed a vector model of patient complexity, identifying interactions among biological, socioeconomic, cultural, environmental, and behavioral forces as health determinants. These forces exert profound influences on processes and outcomes of care for chronic medical conditions.

The application of data management and artificial intelligence tools to support hospital decision-making processes is not new. Moreno et al. (1999), for example, presented a simulation tool that allowed virtual societies such as hospitals to be implemented. Their approach allows to implement a patient-centered simulation tool. Groothuis, Merode, and Hasman (2001) demonstrated that discrete event simulation techniques could be used to optimize the use of catheterization capacity and validated the simulation package called MedModel. In 2002, Walczak, Pofahl, and Scorpio (2002) reported the use of various supervised learning neural network training methods to determine the best possible modeling paradigm for the medical domain problem of predicting a patient's length of stay and severity of injury/illness. Sloane, Liberatore, Nydick, Luo, and Chung (2003) used an analytical hierarchy process to facilitate the selection of neonatal ventilators. In 2010, Herasevich, Pickering, Dong, Peters, and Gajic described the development and implementation of the Multidisciplinary Epidemiology and Translational Research in Intensive Care (METRIC) Data Mart, which is an 
informatics infrastructure for syndrome surveillance, decision support, reporting and modeling of critical illnesses. Chanamool \& Naenna (2016) proposed a Fuzzy failure mode and effects analysis (FMEA) for prioritization and assessment of failures in a hospital emergency department. Nazari, Fallah, Kazemipoor, and Salehipour (2018) developed a Fuzzy Analytic Hierarchy Process (AHP) and Fuzzy Inference System to assess the condition of patients being investigated for heart diseases. Most recently, Wright and Sittig (2019) have developed a clinical decision-making model for pharmacies.

\section{The Four Cs}

Table 2. The 4 C's and their attributes

\begin{tabular}{llll}
\hline Confidence & Complexity & Capability & Customer \\
\hline Condition of patient & Planning & Management of resources & Value \\
Process & Integration & Quality of services & Criticality \\
Uncertainty of action & Coordination & $\begin{array}{l}\text { Demand variability and } \\
\text { uncertainty }\end{array}$ & $\begin{array}{l}\text { Skill Set and Hospilance } \\
\text { Culture Cost }\end{array}$ \\
& Collaboration & Sustainability & \\
& & &
\end{tabular}

Table 2 shows the 4 Cs- complexity, confidence, capability, customer, and their attributes. These parameters, which as gleaned from the literature form the basis of decision-making in a hospital, are discussed below.

\subsection{Confidence}

A hospital constantly deals with cases that vary in nature, routine responses, and urgency. The arrival of new emergency cases can stop previously planned activities, and previously planned activities can impede the acceptance of emergency cases. The confidence of patient outcome is one variable that guides patient acceptance and flows (Institute of Medicine Committee on the Future of Emergency Care in the US Health System. 2006). For each patient, the severity of the illness must be assessed in order to discern the process and time required for treatment. With the help of appropriate classification systems, patients with varied conditions can be categorized, and appropriate medical care can be provided to them. In 1971, Hurtado and Greenlick reported a disease classification system for analysis of medical care utilization (Hurtado \& Greenlick, 1971). Because medical decision-making may be particularly prone to error because of the complexity, urgency, and uncertainty inherent in clinical situations (Redelmeier \& Shafir, 1995), along with a normal classification system, there must also be a mechanism to deal with uncertainty, both in terms of demand and patient outcome. The term "confidence" pertains to the hospital's confidence in correctly and quickly assessing both the process of treatment and any underlying uncertainty.

\subsection{Complexity}

Patients in hospitals need various services for their treatment, from admission procedures to diagnostic tests to treatments and therapies. "Complexity" refers to this aspect of a patient's experience. Every patient goes through a complex web of departments and activities in a hospital. The smoothness of a patient's progress through this web is determined by the planning, integration, coordination, and collaboration among different departments. According to Bardram and Bossen (2005), a set of coordinative tools, such as plans, schemes, procedures, templates, and even physical whiteboards, is designed to provide order and to coordinate the multiple work processes of any hospital. With the growth of managed care has come to a trend toward integrating more services and providers into a coordinated system of care (Wang, Wan, Clement and Begun, 2001). In addition, the advent of managed care has brought significant changes in the method and locus of provider payment as well as more disputes over charges for services. Indeed, the need for improved quality and reduced cost underlies current visions for health care reform.

Faraj and Xiao, while studying knowledge-based coordination in a trauma center in 2006, found that expertise coordination practices were needed to manage evolving skill and knowledge interdependencies during treatment of a single patient and between treatments of different patients.

\subsection{Capability}

According to Ferrera, Cebada, and Zamorano (2014), quality in health care services reflects the capacity of medical staff to diagnose and treat medical problems. More specifically, the capability of a hospital includes the available services and resources, both physical, such as beds, medical instruments, laboratories, and testing equipment, and human, including doctors, nurses, technicians, and multiple other staff members. A hospital's resources must be efficiently and effectively planned and managed, with particular emphasis on inpatient beds, operating theatres, hospital workforce, and expensive 
critical care resources (Harper, 2002) in order to sustainably serve incoming demand (i.e. planning elective patient arrivals) without compromising the quality of service. Mechanisms must be in place to ensure that people, irrespective of their ability to pay, will have continued access to prompt, technologically current, competent, and compassionate health care that addresses the full range of their health needs (Guyatt, Yalnizyan and Devereaux, 2002) in a sustainable way.

\subsection{Customer}

Patients come from different socio-economic backgrounds that should be factored into decision-making about treatment. An essential goal of each medical encounter is for the doctor and patient to develop a shared understanding (what Safford, Allison, and Keife have termed "congruence") about realistically attainable healthcare goals (2007). Attributes inherent in this category include value, criticality, surveillance, and cost. A 2010 study described nine aspects of value, including cost, equipment, physician background, physician care, environment, timing arrangement, relationship, brand image, and additional value, to construct an objective network for customer value (Lee, 2010). Criticality, or risk criticality, is determined by the likelihood of a patient safety risk event occurring as well as the severity of its impact. Once criticality for a patient risk event has been determined, the next step is to establish its detectability (Cupryk, 2011). According to Gaynes et al., (2001) surveillance is immediate and strong mandatory reporting of medical errors and voluntary reporting of other adverse health events. According to Taheri, Butz, and Greenfield (2000), cost, including issues of hospital cost containment, cost reduction, and lower-cost alternative care delivery systems, is an important concern for healthcare providers, payers, employers, and policymakers throughout the United States. Kuo and Goodwin, in 2011, studied the relationship of hospitalist care with hospital length of stay, hospital charges, medical utilization, and Medicare costs after discharge. They concluded that decreased length of stay and hospital costs associated with hospitalist care are offset by higher medical utilization and costs after discharge.

\section{Hospital Environment Evaluation: Graph-Theoretic Approach}

The 4-C Model provides a mathematical model of a hospital's environment that encompasses all four interacting factors-confidence, complexity, capability, and customer--using a graph-theoretic approach based on the digraph approach to TQM evaluation of an industry reported by Grover et al. (2004). TQM is a management approach to detect and reduce errors. In the graph-theoretic model, abstract information regarding the system is represented by the edges of a directed graph, or digraph, which depict how one factor affects the others. The digraph makes a matrix model that is useful for computer processing. The permanent value of a multinomial developed from a matrix can represent an environment using a single number/index (Grover et al., 2004). In this study, the net effect of different factors on the hospital environment is estimated in terms of a single numerical index by quantification of different factors--confidence, complexity, capability, and customer--and their interdependencies. Each factor has attributes in itself that interact with each other and contribute to quantifying the factor. How these attributes and their interactions are quantified depends on the organization itself and needs to be determined by the hospital's experts. This analysis, however, is at a subsystem level, and a comprehensive study of all such combinations is outside the scope of this paper.

\subsection{Digraph Matrix, Permanent Value Multinomial and Quantification}

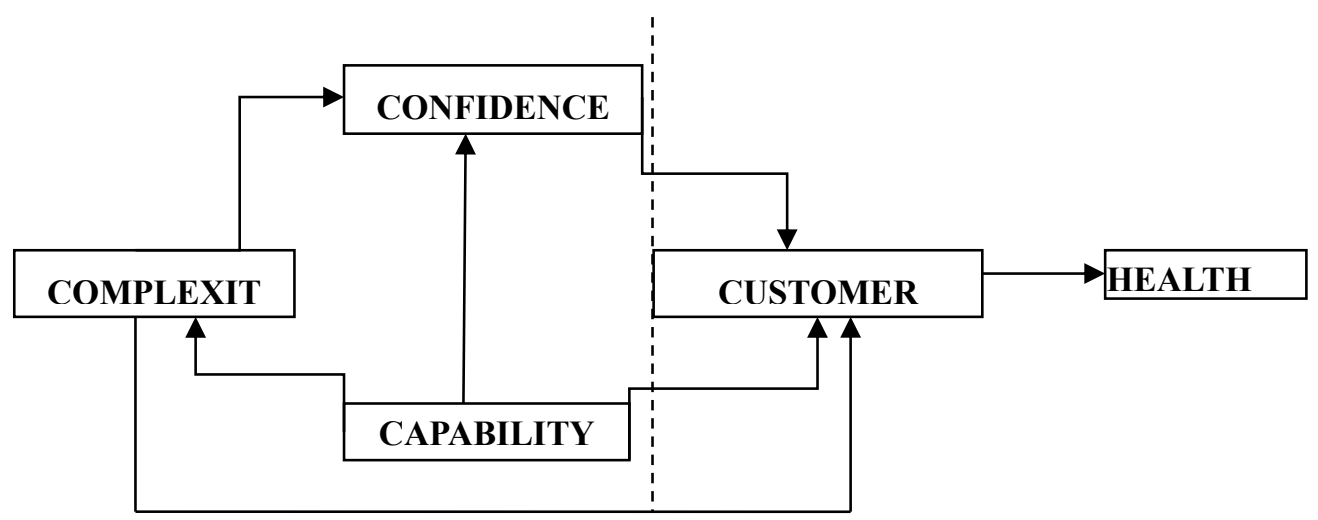

Figure 1. Schematic Diagram of Health Care System

Figure 1 shows a schematic diagram of the healthcare system comprising the $4 \mathrm{Cs}, \mathrm{C}_{1}$ (confidence), $\mathrm{C}_{2}$ (complexity), $\mathrm{C}_{3}$ (capability), and $\mathrm{C}_{4}$ (customer), respectively. The factors and their interdependencies are presented in terms of the nodes and edges of the digraph. Directions are assigned to the edges in the digraphs. In a digraph environment, the measure of characteristics or factors $\left(\mathrm{C}_{\mathrm{i}}\right.$ 's) is represented through its nodes, while the edges show the dependence of factors $\left(\mathrm{C}_{\mathrm{ij}}{ }^{\prime} \mathrm{s}\right)$. $\mathrm{C}_{\mathrm{ij}}$ indicates how much the $j^{\text {th }}$ factor is dependent on the $i^{\text {th }}$ factor. In the digraph, $\mathrm{C}_{\mathrm{ij}}$ is represented as a directed edge from node $i$ to node $j$ (Grover et al., 2004). 


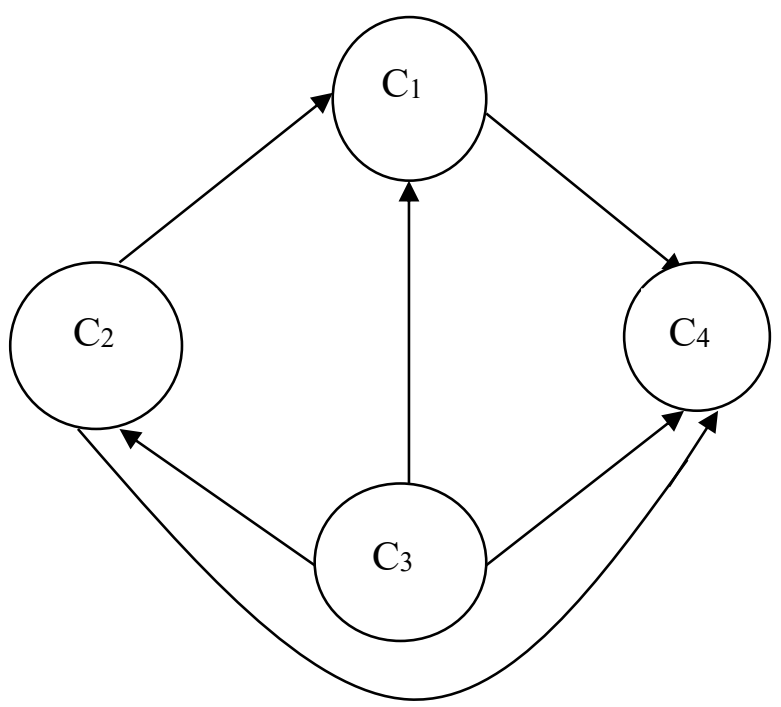

Figure 2. Digraph for the System, the 4C Model

Figure 2 shows a digraph of the 4-C model. According to the digraph, out of the $4 \mathrm{Cs}$, the most influential for the conduciveness for decision-making is capability $\left(\mathrm{C}_{3}\right)$ because capability has repercussions for all of the other factors. If a hospital has a lack of resources (an aspect of capability), many other decisions will be out of its control. For example, if resources such as beds are few or not adequately managed, the hospital will not be confident of taking up more cases. Similarly, if the workforce isn't adequately competent (another aspect of capability), there will be greater uncertainty about taking appropriate actions. Similarly, efficient management is simpler, with less built-in complexity. If a hospital lacks the leadership to form plans, issue guidelines, and provide infrastructure for the integration and collaboration of different departments, then activities in the hospital may be chaotic or confused. Finally, it is the capability of the hospital as reflected by its reputation that attracts and retains customers $\left(\mathrm{C}_{4}\right)$. If the infrastructure and staff are not up to standards, patients will not trust it enough to seek treatment there. Thus, the capability has the most direct impact on all aspects of the patient experience.

Complexity $\left(\mathrm{C}_{2}\right)$ governs how confident $\left(\mathrm{C}_{1}\right)$ a hospital is in accepting and tackling cases. When the hospital takes a patient, first the patient is diagnosed, and then a treatment is prescribed; the treatment course is scheduled and incorporated into the plans for the different departments, and then the patient proceeds through the web of activities. If there is a lack of coordination and collaboration, the hospital will not be so confident in taking up new cases, as it may be uncertain whether the system is competent to handle the process. Thus, confidence strongly affects the customer $\left(\mathrm{C}_{4}\right)$. Complexity $\left(\mathrm{C}_{2}\right)$ also affects the customer $\left(\mathrm{C}_{4}\right)$ directly. The smoother the flow of the patient's management between departments and facilities, the greater the value received by the patient and the better the patient experience is.

\subsection{Matrix}

To render the information in the digraph more convenient for computer processing, the digraph is converted into a matrix that sets up a numerical expression for the elements. Let us assume a digraph of $\mathrm{n}$ factors forms an $n^{\text {th }}$ order symmetric $(0,1)$ matrix $\mathrm{A}=\left[C_{i j}\right]$. The elements of rows and columns in the matrix indicate interactions among factors; for example, the interaction of the $i^{\text {th }}$ factor with the $j^{\text {th }}$ factor is denoted by $C_{i j}$ :

$$
C_{i j}=1 \text {; if factor } \mathrm{i} \text { is connected to factor } \mathrm{j} \quad=0 \text {, otherwise }
$$

Generally, $C_{i j} \neq C_{j i}$ as the factors are directional, and $C_{i i}=0$ as a factor is not interacting with itself. The matrix is square and non-symmetric and is analogous to the adjacency matrix in graph theory (Grover, et al., 2004).

$$
A=\left(\begin{array}{cccc}
0 & 0 & 0 & 1 \\
1 & 0 & 0 & 1 \\
1 & 1 & 0 & 1 \\
0 & 0 & 0 & 0
\end{array}\right)
$$

Matrix Representation of digraph in Figure 2 


\subsection{Variable Permanent Matrix}

Both digraph and matrix representations are dependent on the labeling of nodes. As they change by changing the labeling of nodes, a permanent function of the matrix (Variable Permanent Matrix) was developed by Grover et al., (2004) to give a unique representation independent of labeling.

$$
\mathrm{C}^{*}=\left(\begin{array}{llll}
C_{1} & 0 & 0 & C_{14} \\
C_{21} & C_{2} & 0 & C_{24} \\
C_{31} & C_{32} & C_{3} & C_{34} \\
0 & 0 & 0 & C_{4}
\end{array}\right)
$$

VPM for the digraph in Figure 2.

\subsection{Permanent Representation}

The variable permanent function (VPF), being the characteristic of the environment of an organization, is a powerful tool for analysis (Grover, et al., 2004). This permanent function is calculated in the same way as a determinant except that the negative sign in the calculation of the determinant is substituted with a positive sign in the variable permanent function. The result of this computational process yields a multinomial. The VPF offers a quantitative environment for the evaluation of an organization by putting numerical values on the $C_{i}$ 's and $C_{i j}$ 's. The values of the $C_{i}$ 's and $C_{i j}$ 's are obtained either analytically or by comparison with ideal cases. This single numerical index indicates the environment of an organization in quantitative terms. The VPF for a 4-factor digraph is given as

$$
\begin{aligned}
\text { Per } \mathrm{C}^{*}=\prod_{i=1}^{4} C_{i}+ & \sum_{i} \sum_{j} \sum_{k} \sum_{l}\left(C_{i j} C_{j i}\right) C_{k} C_{l}+\sum_{i} \sum_{j} \sum_{k} \sum_{l}\left(C_{i j} C_{j k} C_{k i}+\left(C_{i k} C_{k j} C_{j i}\right) C_{l}\right. \\
& +\sum_{i} \sum_{j} \sum_{k} \sum_{l}\left(C_{i j} C_{j i}\right)\left(C_{k l} C_{l k}\right)+\sum_{i} \sum_{j} \sum_{k} \sum_{l}\left(C_{i j} C_{j k} C_{k l} C_{l i}+C_{i l} C_{l k} C_{k j} C_{j i}\right)
\end{aligned}
$$

The significance of the equation is the following:

- All $n$ unconnected elements of a digraph are grouped together in the first group of the equation

- Self-looping comes in the second grouping, but in the present case, self-looping is absent, so this group's value is zero.

- In the third grouping, each term shows a set of two-element digraph (i.e., $C_{i j} C_{j i}$ ) interdependence characteristics of $i$ and $j$ and the remaining $\mathrm{N}-2$ unconnected elements.

- In the fourth grouping, each term shows a set of three-element digraph (i.e., $C_{i j} C_{j k} C_{k} i$ or its pair (i.e., $C_{i k} C_{k j} C_{j i}$ ) interdependence characteristics of $i, j, k$, and the remaining $\mathrm{N}-3$ unconnected elements.

- There are two subgroups in the fifth grouping. The first subgroup consists of the product of two elements $C_{i j} C_{j i}$ and $C_{k l} C_{l k}$. The second subgroup represents all four elements (i.e., $C_{i j} C_{j k} C_{k l} C_{l i}$ ) or its pair (i.e., $C_{i l} C_{l k} C_{k j} C_{j i}$ )

4.5 Quantification of $C_{i}$ 's and $C_{i j}$ 's

For evaluation of a VPF, numerical values of the $C_{i}$ 's and $C_{i j}$ 's (i.e., the diagonal and off-diagonal elements of the VPM) are required. To obtain the values of the diagonal elements (i.e., the $C_{i}$ 's), each $C_{i}$ is considered as a subsystem and the graph-theoretic approach is used in each subsystem. These values can be assigned by local hospital experts familiar with their particular hospitals.

Table 3. Quantifiers for factors suggested by Grover et al., (2004)

\begin{tabular}{lll}
\hline S. No. & Qualitative Measure of a Particular Factor & Assigned Quantifier \\
\hline 1 & Exceptionally Low & 1 \\
2 & Very Low & 2 \\
3 & Low & 3 \\
4 & Below Average & 4 \\
5 & Average & 5 \\
6 & Above Average & 6 \\
7 & High & 7 \\
8 & Very High & 8 \\
9 & Exceptionally High & 9 \\
\hline
\end{tabular}


Table 4. Quantifiers for interdependencies suggested by Grover et al., (2004)

\begin{tabular}{lll}
\hline S. No. & Qualitative Measure of Interdependencies & $\mathrm{C}_{\mathrm{ij}}$ \\
\hline 1 & Very Weak & 1 \\
2 & Weak & 2 \\
3 & Medium & 3 \\
4 & Strong & 4 \\
5 & Very Strong & 5
\end{tabular}

Quantifiers for factors and quantifiers for interdependences are given in Table 3 and Table 4, respectively.

\section{Methodology}

The graph-theoretic procedure to determine the conduciveness of a hospital to decision-making is as follows:

- First, the various factors affecting the hospital environment are identified by local hospital experts, and the attributes affecting those factors are listed.

- A digraph showing the interdependencies of the attributes upon the factors is generated.

- A variable permanent matrix at the system level is developed.

- The digraph of the subsystem is created, and according to the subsystem digraph, a permanent matrix is prepared.

- Finally, the subsystem permanent function is calculated similarly to how the other $C_{i}$ 's are calculated and considering the attributes affecting each $C_{i}$ or $C_{i j}$; that is, the off-diagonal elements indicating interdependencies are assigned numerical values.

- This dependence between the elements at the system level or subsystem level cannot be measured directly (Grover et al., 2004). So, these values can be assigned by experts. However, Grover et al., (2004) provided suitable values in their TQM environment implementation, and similar values have been used in the example presented in this paper.

- The variable permanent function shows the conduciveness level of the hospital.

\subsection{Case Study}

To demonstrate the proposed methodology, a case study was conducted in a local hospital in Rajasthan, India. Each factor, along with its attributes, was quantified using a field survey, by a panel of five health experts consisting of two senior management experts and three senior doctors. It should be noted that this example case study depicted only one set of interactions. Different such scenarios could exist and be quantified using this model. Experts can compile a comprehensive database and each different combination could lead to a different environment in the hospital, thus forming a scale. For each environment, there could be a standard set of guidelines for the various decisions made in a hospital. Once this is achieved, the 4-C model could provide a unique decision support tool that considers the various interdependencies and interactions of the factors affecting a hospital's working environment 
Table 5. Set of indicators per factor attribute

\begin{tabular}{|c|c|c|}
\hline Factor & Attribute & Criterion \\
\hline \multirow{6}{*}{ Confidence } & $\begin{array}{l}\text { Condition of patient } \\
\left(\mathrm{F}_{1}\right)\end{array}$ & Number of diagnoses bad interpreted \\
\hline & & Number of admissions that do not have a fixed criterion \\
\hline & Process $\left(\mathrm{F}_{2}\right)$ & \\
\hline & & Number of patients that do not fulfil the ideal cycle time \\
\hline & $\begin{array}{l}\text { Uncertainty of } \\
\text { action }\left(F_{3}\right)\end{array}$ & Number of biases identified. \\
\hline & $\begin{array}{l}\text { Uncertainty } \quad \text { of } \\
\text { outcome }\left(\mathrm{F}_{4}\right)\end{array}$ & Number of inaccurate of treatments selected \\
\hline \multirow{4}{*}{ Complexity } & Planning $\left(\mathrm{P}_{1}\right)$ & Lack of accomplishment percentage of planning versus execution \\
\hline & Integration $\left(\mathrm{P}_{2}\right)$ & $\begin{array}{l}\text { Number of physicians are not trained in prehospital and hospital } \\
\text { plans }\end{array}$ \\
\hline & Coordination $\left(\mathrm{P}_{3}\right)$ & Percentage of bottlenecks or obstacles in patient flow \\
\hline & Collaboration $\left(\mathrm{P}_{4}\right)$ & Unbalanced distribution of responsibility in a team \\
\hline \multirow{8}{*}{ Capability } & Management of & Percentage of incidents where there are shortages. \\
\hline & resources $\left(B_{1}\right)$ & Inventory \\
\hline & $\begin{array}{l}\text { Quality of services } \\
\left(\mathrm{B}_{2}\right)\end{array}$ & Percentage of customers who perceive they received a bad service. \\
\hline & $\begin{array}{l}\text { Demand variability } \\
\text { and uncertainty }\left(\mathrm{B}_{3}\right)\end{array}$ & Coefficient of variation of the demand \\
\hline & & \\
\hline & Skill Set and & Percentage of staff members are not trained in process design \\
\hline & $\begin{array}{l}\text { Hospital Culture } \\
\left(\mathrm{B}_{4}\right)\end{array}$ & Percentage of staff member who does not have ownership sense \\
\hline & Sustainability $\left(\mathrm{B}_{5}\right)$ & $\begin{array}{l}\text { Historical behavior of implemented continuous improvement } \\
\text { techniques. How often such techniques do not remain working. }\end{array}$ \\
\hline \multirow{4}{*}{ Customer } & Value $\left(\mathrm{T}_{1}\right)$ & Bad value customer perception facing offered value \\
\hline & Criticality $\left(\mathrm{T}_{2}\right)$ & Number of adverse incidents \\
\hline & Surveillance $\left(\mathrm{T}_{3}\right)$ & Number of deficiencies did not identify by the surveillance system \\
\hline & $\operatorname{Cost}\left(\mathrm{T}_{4}\right)$ & Ratio of cost \\
\hline
\end{tabular}

Table 5 shows the factors, attributes, and their criteria. As mentioned before, each panel of experts may assign values to every factor's attributes based not only on the panel's expertise but also on performance indicators that each such institution might have. It is important to note that this may vary from one institution to another because every organization has its own key performance indicators. In order to illustrate, a set of indicators on which the experts could base their evaluations is shown in Table 5. To simplify the analysis, all the attributes must have the same improvement direction. That is, all of them are better to an extent when all of them are increased or all of them are decreased. In this example, the smaller the value of the quantifiers assigned to the attributes, the better is the conduciveness of the system. 
Table 6. Elements of 4C'S and system VPM

\begin{tabular}{|c|c|c|c|c|c|c|c|c|c|}
\hline \multicolumn{2}{|c|}{ Confidence } & \multicolumn{2}{|c|}{ Complexity } & \multicolumn{2}{|c|}{ Capability } & \multicolumn{2}{|c|}{ Customer } & \multicolumn{2}{|c|}{ System } \\
\hline $\begin{array}{l}\text { VPM } \\
\text { Elemen } \\
t\end{array}$ & $\underset{r}{\text { Quantifie }}$ & $\begin{array}{c}V P M \\
\text { Elemen } \\
\quad t\end{array}$ & $\underset{r}{Q \text { Quantifie }}$ & $\begin{array}{c}V P M \\
\text { Elemen } \\
\quad t\end{array}$ & $\underset{r}{Q \text { Quantifie }}$ & $\begin{array}{c}V P M \\
\text { Elemen } \\
\quad t\end{array}$ & $\begin{array}{c}\text { Quantifie } \\
r\end{array}$ & $\begin{array}{c}V P M \\
\text { Elemen } \\
t\end{array}$ & $\begin{array}{c}\text { Quantifie } \\
r\end{array}$ \\
\hline$F_{l}$ & 8 & $P_{1}$ & 5 & $B_{1}$ & 8 & $T_{I}$ & 9 & $C_{1}$ & 3.49 \\
\hline$F_{2}$ & 7 & $P_{2}$ & 6 & $B_{2}$ & 8 & $T_{2}$ & 7 & $C_{2}$ & 3.16 \\
\hline$F_{3}$ & 4 & $P_{3}$ & 8 & $B_{3}$ & 5 & $T_{3}$ & 6 & $C_{3}$ & 3.95 \\
\hline$F_{4}$ & 6 & $P_{4}$ & 6 & $B_{4}$ & 7 & $T_{4}$ & 8 & $C_{4}$ & 3.48 \\
\hline$F_{12}$ & 5 & $P_{12}$ & 3 & $B_{5}$ & 4 & $T_{14}$ & 5 & $C_{21}$ & 3 \\
\hline$F_{13}$ & 3 & $P_{13}$ & 4 & $B_{12}$ & 4 & $T_{21}$ & 3 & $C_{14}$ & 4 \\
\hline$F_{14}$ & 4 & $P_{32}$ & 4 & $B_{15}$ & 4 & $T_{23}$ & 4 & $C_{24}$ & 3 \\
\hline$F_{23}$ & 3 & $P_{43}$ & 4 & $B_{25}$ & 2 & $T_{24}$ & 3 & $C_{31}$ & 4 \\
\hline$F_{34}$ & 4 & & & $B_{31}$ & 4 & $T_{34}$ & 3 & $C_{32}$ & 4 \\
\hline$F_{42}$ & 3 & & & $B_{32}$ & 2 & & & $C_{34}$ & 5 \\
\hline & & & & $B_{35}$ & 2 & & & & \\
\hline & & & & $B_{42}$ & 5 & & & & \\
\hline & & & & $B_{45}$ & 3 & & & & \\
\hline
\end{tabular}

Table 6 shows the value of the quantifiers assigned by the XYZ hospital experts. These values will be used in subsequent sections for calculation.

\subsubsection{Confidence}

The attributes of confidence are the following: the condition of the patient, the process, the uncertainty of the outcome, and the uncertainty of action. For the sake of simplicity, they have been renamed $F_{1}, F_{2}, F_{3}$, and $F_{4}$, respectively.

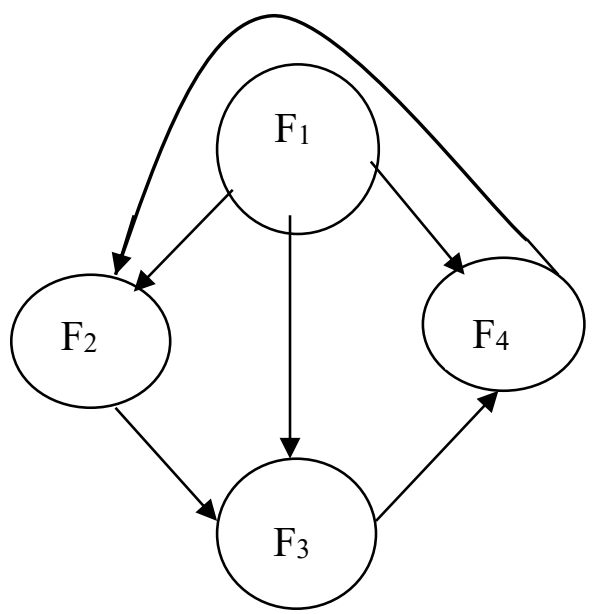

Figure 3. Confidence Digraph

Figure 3 shows the digraph of confidence with those four attributes. The direction of this digraph was obtained from the following considerations. A patient's condition is important in determining a hospital's confidence in dealing with any case. Bornstein and Emler, in 2001, reported that when making a diagnosis, doctors tend to order tests that improve the probability of the initial tentative diagnosis. Since confirmation bias is present, a patient with a set of symptoms directly indicating a particular condition will cause the hospital to schedule its process accordingly. Thus, the symptoms and condition of the patient have a powerful influence on the confidence. However, often because of the individual culture and policies of the hospital, the same symptom may be interpreted differently, and different diagnoses can be made. In the case of a different diagnosis, different treatment will be prescribed, and the schedules and acceptance of new cases will be done accordingly. The "fuzziness" of the symptom and the consequent (mis)interpretation may lead to a completely different treatment, and thus a different process. Therefore, it can be safely assumed that the condition of the patient 
affects the process very strongly. Once a process suitable for the patient is recognized and charted, the hospital can be more confident of how many new cases can be taken. For example, elective surgeries can be scheduled with much more ease. Thus, the process has a strong influence on the confidence of the hospital.

Thiery et al. (2005) reported that oncologists and intensivists rationally reserve ICU admission for cancer patients with potentially reversible medical conditions and a "reasonable prospect of substantial recovery." This finding suggests that the criteria for admission in the hospital are, to a certain extent, fixed. Thus, the outcome is a factor, though not a major one, in determining the confidence of the hospital in dealing with new cases. Therefore, we can say that uncertainty of outcome has an average influence on confidence.

The findings of Nadkarni et al. (2006) suggest that the condition of the patient has a moderate, if not high, influence on the outcome since it can be used to predict what the result may or may not be. Treatment choices can be affected by the possible outcomes of various treatments (Bornstein and Emler, 2001). However, at least one treatment (or no treatment) has to be selected. Thus, the uncertainty of an outcome has a reasonable effect on the uncertainty of action. McNeil et al. conducted a study in 1982 in which participants were asked to assume that they had lung cancer. Two alternative treatments (surgery and radiation) were offered to them, along with their probability of survival and life-expectancy data. A "framing" bias was observed; i.e., the individuals made decisions depending on how the potential benefits and harms of the alternative treatments were presented. Such a conclusion is crucial to the process of treatment, so the uncertainty of action, which was prone to biases, affected the process strongly. Thus, the uncertainty of action plays a somewhat average role in determining confidence since ultimately, some action must be taken. If so, the confidence of the organization, quantifiers, and VPM will be found. The VPM values from the digraph are the following:

$$
\left(\begin{array}{cccc}
F_{1} & F_{12} & F_{13} & F_{14} \\
0 & F_{2} & F_{23} & 0 \\
0 & 0 & F_{3} & F_{34} \\
0 & F_{42} & 0 & F_{4}
\end{array}\right)
$$

\section{Matrix 1 VPM for Confidence}

The values taken from Table 6 are $F_{1}=8, F_{12}=5, F_{13}=3, F_{14}=4, F_{2}=7, F_{23}=3, F_{3}=4, F_{34}=4, F_{42}=3, F_{4}=6$. Substituting the values in Matrix 1 results in

$$
\left(\begin{array}{llll}
8 & 5 & 3 & 4 \\
0 & 7 & 3 & 0 \\
0 & 0 & 4 & 4 \\
0 & 3 & 0 & 6
\end{array}\right)
$$

\section{Matrix 1 VPM for Confidence}

The value of the VPF thus calculated will be 3072 by substituting the values in Equation 1. It should be noted that a more confident organization will have a different value.

\subsubsection{Complexity}

The attributes of complexity are planning, integration, coordination, and collaboration. For the sake of simplicity, they have been renamed $P_{1}, P_{2}, P_{3}$, and $P_{4}$, respectively. 


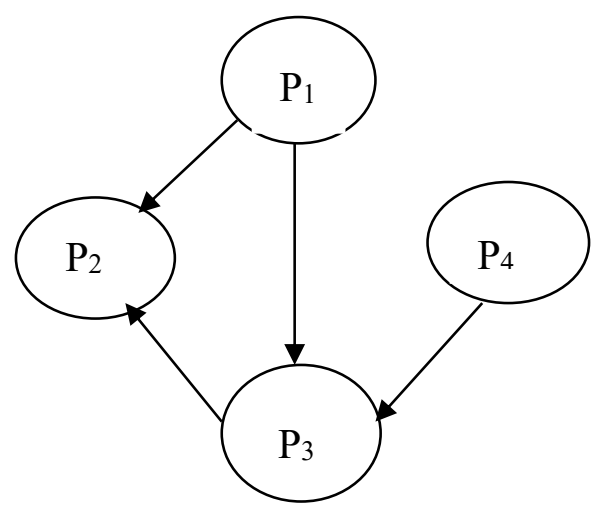

Figure 4. Complexity Digraph

Figure 4 shows the digraph of confidence having all four attributes. The direction of this digraph is obtained from the following discussion. In a hospital, the number of patients in an operation theatre must be planned for a specified period. The operating theatre consists of various operating rooms, along with recovery rooms. Each patient needs a particular surgical procedure, which defines the human (surgeon) and material (equipment) resources to use. The duration of each intervention and each loaded operating room are scheduled individually to synchronize the various human and material resources used (Guinet and Chaabane, 2003). Thus, planning is required to achieve greater coordination of human and material resources. So, it may be said that planning strongly affects coordination.

In a mass-casualty incident, pre-hospital and hospital care services must be integrated because limited resources are available for multiple casualties. In order to obtain optimal care for mass casualties, each emergency physician must be aware of both pre-hospital and hospital plans. By taking part in the planning phase, the emergency physician will ensure that the patients receive the best care using the available resources; however, planning is just the first step towards integration. Hence, planing has some, but not a very strong influence on integration; coordination has a greater influence. In 1993, Conrad reported that optimal personal health would be obtained by vertical integration in health care that consisted of the coordination of inputs (equipment, supplies, human resources, information, and technology) and intermediate outputs (preventive, diagnostic, acute, chronic, and rehabilitative services). Thus, coordination affects integration quite strongly.

Increasing costs of health care and rapid knowledge growth have led to greater collaboration among health care professionals in order to share knowledge and skills (Patel et al., 2000). Patel et al., (2000) found that the distribution of responsibilities in a team reduces unnecessary and redundant interaction as well as the cognitive load on individuals. With less cognitive load, the physicians can pay more attention to coordination. Thus, collaboration strongly affects coordination in a hospital.

It can be seen from the above discussion that while planning does cause a better organization of the complex web of activities in a hospital (or complexity), it is not the most important factor since everything depends on how well the implementation is carried out. Hence, planning has somewhat of a neutral effect on complexity. However, the implementation (or coordination as we are terming it here), has a powerful influence on complexity because without it, schedules will not run on time, and there is likely to be an underutilization of resources. Collaboration between partners in complex coordinated care is essential, but planning is also required to make it work. Hence, collaboration has an aboveaverage influence on complexity. While horizontal and vertical integration has helped organizations, they are not the sole solution to complex flow problems. Therefore, only an above-average effect is exerted by complexity. The VPF value will be 1440. The elements of complexity's VPM are given as shown in Table 6.

Matrix 2 VPM for Complexity

$$
\left(\begin{array}{llll}
5 & 3 & 4 & 0 \\
0 & 6 & 0 & 0 \\
0 & 4 & 8 & 0 \\
0 & 0 & 4 & 6
\end{array}\right)
$$

\subsubsection{Capability}

The attributes of capability are resource management, service quality, demand variability, demand uncertainty, skill set, hospital culture, and sustainability. For the sake of simplicity, they have been renamed $B_{1}, B_{2}, B_{3}, B_{4}$ and $B_{5}$, respectively. 


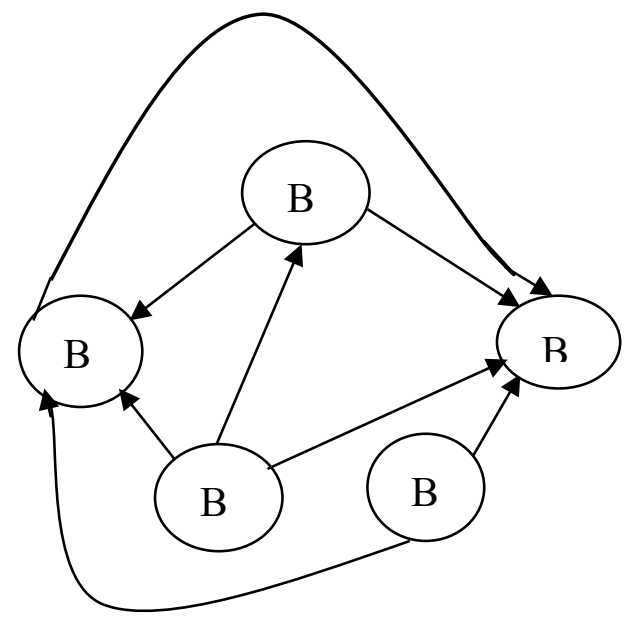

Figure 5. Capability Digraph

Figure 5 shows the digraph of capability with all its four attributes. The direction of this digraph is determined from the following discussion. Patients are the customers of health care services, which are driven by capacity. Thus, capacity resource management affects the type of service offerings as well as customer satisfaction, cost, and quality of service. In response to the current health service environment, the linkage between capacity management and performance is underscored. Successful health service organizations tend to have a superior ability to manage their capacity resources (Li and Benton, 2003). Thus, effective management of resources has a very high influence on the capability of a hospital. It also strongly affects the quality of services offered in the hospital.

Clinical quality and customer satisfaction are measures of quality. Clinical quality consists of proper equipment, timely treatment, adequate services, and the ability to meet acceptable industry practice standards. Customer satisfaction is increased by identifying customer needs and areas for improvements as determined by customers' feedback (Li and Benton, 2003). Hospital patients receive a specific impression regarding any hospital within a fraction of time. A good hospital's image is built by a patient's trust in the prescribed treatment and by knowledge of the hospital's staff. These can improve the patient/consumer's tendency to select that hospital in the future (Kim et al., 2008). It is also a reflection of the hospital's effectiveness. Thus, quality is a high indicator of capability.

Like other industries, the healthcare industry experiences variability in demand. Since changes in demand prompt hospital administrations to manage their resources, demand variability may be said to strongly affect the management of resources. However, hospitals are expected service quality regardless of demand fluctuations even though decline in quality may somewhat affect an increase in demand. Hence, demand only weakly affects the quality of services. Moreover, quality of service and cost of service require effective staff members, as proficient and knowledgeable staff design processes more efficiently and provide services that better satisfy customers' needs and expectations. To retain health care service quality while containing costs, hospitals have begun to emphasize human resource development (Li and Benton, 2003). A good workforce thus speaks highly of the capability of the hospital.

Another attribute of capability is organizational sustainability; seen from a complexity theory perspective, sustainability is a continuous process of co-evolution with a changing environment (Mitleton-Kelly, 2011). A sustainable system works changes by changing set conditions and building new structures as need requires. While organizational sustainability is an indication of long-term capability that should be considered in making current decisions, it isn't an important factor in decision-making. Thus, it has a weak influence on the capacity of present decision-making. Leadership and creation of an enabling environment are necessary but not sufficient, as changes should be embedded within the organizational culture through a different way of working, relating, and thinking (Mitleton-Kelly, 2011). Thus, the skill set of a workforce plays a moderate role in creating sustainability. Sustainability also means being prepared for future demands; hence, demand variability has a slightly less-than-average effect on sustainability. However, it is highly dependent on how well resources are managed in the hospital and slightly dependent on the maintenance of the quality of services. The VPF value is 8960. See Table 6 for the capability VPM elements. 


$$
\left(\begin{array}{ccccc}
8 & 4 & 0 & 0 & 4 \\
0 & 8 & 0 & 0 & 2 \\
4 & 2 & 5 & 0 & 2 \\
0 & 5 & 0 & 7 & 3 \\
0 & 0 & 0 & 0 & 4
\end{array}\right)
$$

\section{Matrix 3 VPM for Capability}

\subsubsection{Customer}

The attributes of customers include the value sought by the customer, the criticality within the hospital, the surveillance thus required, and the cost that the customer must pay. For the sake of simplicity, customer attributes are here termed $T_{l}$, $T_{2}, T_{3}$, and $T_{4}$, respectively.

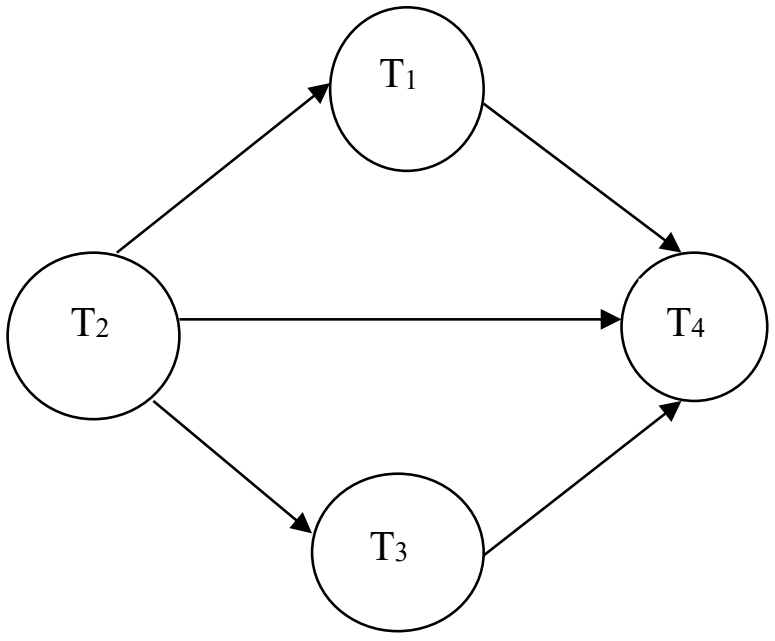

Figure 6. Customer Digraph

Figure 6 shows the digraph of customer having all four attributes. The direction of this digraph is obtained from the following discussion. Value is any activity for which a customer is willing to pay. In a hospital setting, the patient is the customer, and the thing for which the customer is willing to pay in this context is good care. According to Sahinoglu and Wool (2014) strategies for quality improvement are aimed at patients' receiving the appropriate care at the right time and place with the appropriate mix of information and available supporting resources. However, healthcare systems are not always designed according to patients' needs. To overcome the flaws of the system, a new patient-centered approach replaces the traditional physician-centric approach with a care team that includes the primary care doctor, psychologist, nurse, and pharmacist, all of whom look after numerous patients in a group setting. A patient will be willing to pay for a service that centers on him or her, and this perception of value is what affects cost. Not only are the patient's needs being taken care of, but there is also a sense of surety that no adverse incident (hospital-borne infection or adverse reaction to a drug) will happen.

Characterizing and assessing the patient-centered quality of care (service) in risky situations or determining how to costoptimize unacceptable risks to a tolerable level within the available budgetary and personnel resources is a complex but essential task (Sahinoglu and Wool, 2014). How susceptible a patient is to such risk can be termed his or her criticality. Thus, a patient's criticality plays a part in determining the definition of value for him or her. To assess this criticality, hospital management need an efficient and appropriate surveillance system, such as Failure mode effects and criticality analysis (FMECA) or automated surveillance. For example, the criticality of a patient's compromised immune system because of treatment with steroids governs which surveillance system should be used. These relationships--value, criticality, and surveillance--all affect the cost to be paid by the patient. In addition, finances are always a significant issue for a patient. Healthcare expenditures continue to rise, and governments are putting pressure on healthcare professionals and patients to become more cost-aware. This attribute of a customer, cost awareness, plays a huge role in determining hospital policies, and in defining its environment. The VPF value is 3024. See the VPM value for the customer in Table 6. 
Matrix 4 VPM for Customer

$$
\left(\begin{array}{llll}
9 & 0 & 0 & 5 \\
3 & 7 & 4 & 3 \\
0 & 0 & 6 & 3 \\
0 & 0 & 0 & 8
\end{array}\right)
$$

\section{Results and Discussions}

Table 7. Permanent VPF values for the subsystems and the system

\begin{tabular}{ccccc}
\hline Confidence & Complexity & Capability & Customer & System \\
\hline 3072 & 1440 & 8960 & 3024 & 151.52
\end{tabular}

Table 7 shows the VPF value of both the subsystem and the system. The subsystem values thus obtained are far too large, compared to the values that will be used for interdependence. Therefore, they may be normalized. Hence, the system level digraph will be as follows and the quantifiers may be as suggested. The diagonal quantifiers are arrived at by taking the logarithmic terms (log (base 10)) (Gurumurthy et al., 2013) of every individual VPF obtained from every VPM of the factors and off-diagonal quantifiers from Table 4. Using these, the VPF value comes out to be 151. The larger value of VPF indicates that the hospital is more conducive.

The foregoing depicts how easy it is for the hospital administration to make crucial decisions in a hospital. If the environment is more conducive, then appropriate decisions will be made more promptly in order to save lives. Every hospital is different from others in terms of confidence, complexity, capability, and customer expectations and service. Thus, each hospital has a different level of conduciveness for clinical decision-making. Very often, the same hospital can face different situations, and accordingly, it will have different conduciveness for decision-making.

This case study is, however, just one possible scenario. There may be several scenarios that need to be examined in order to create a database for decision support. Comparing all of these scenarios would yield a general idea about either the conduciveness among different hospitals or its evolution within one hospital. Over time, such a process would provide a basis for the hospital system to assess itself and improve. If this value or index is calculated for different scenarios, a database and a scale can be created. For each point on this scale of ease of clinical decision-making, industry experts can provide a set of guidelines to be followed regarding different aspects of the hospital each state. Based on the range in which a particular hospital's score lies, it will be given a set of specific guidelines according to which the hospital administration will make every decision. The guidelines can range from being related to patient admittance to diagnostic and treatment processes.

Matrix 5 VPM for the system

$\left(\begin{array}{cccc}3.49 & 0 & 0 & 4 \\ 3 & 3.16 & 0 & 3 \\ 4 & 4 & 3.95 & 5 \\ 0 & 0 & 0 & 3.48\end{array}\right)$

A hospital's working environment depends on the confidence of the outcome for a case, the complexity involved, the capability of the staff, and the needs of the customer. These can be integrated to form an indicator for medical decisionmaking using the 4-C model, which is based on a graph-theoretic approach that evaluates the environment of an organization using a single numerical index. This numerical index takes into consideration the effects of the four factors as well as their interdependencies. The graph-theoretic approach allows for both qualitative and quantitative modeling, and is flexible enough to permit the inclusion of new factors and to generate alternative environments. Using these properties, experts in healthcare management can generate different combinations of interactions among the factors affecting the hospital's working environment and assign appropriate quantifiers to them. This creates a database and a conduciveness scale which could provide a foundation for setting guidelines customized to the scenario in a hospital. A hospital can have different scenarios depending on how the attributes like uncertainty, complexity, capability, and patient risk affect each other; each such scenario needs a different set of actions to take place for a similar problem. Describing each such scenario is out of the scope of this paper. However, we described and measured one such scenario and other scenarios can be measured on the same lines. With this, a scale of the conduciveness of the environment for decisionmaking can be created and this scale can be used by hospitals and their staff to make crucial decisions. To implement the 
methodology systematically will allow the hospitals to identify the enablers for a conducive environment as well as roadblocks of this environment. Thus, this paper demonstrates how the working environment of a hospital can be quantified for a better understanding of clinical decision-making.

\section{References}

Abdel-Basset, M., Manogaran, G., Gamal, A., \& Chang, V. (2019). A novel intelligent medical decision support model based on soft computing and IoT. IEEE Internet of Things Journal, 7(5), 4160-4170. https://doi.org/10.1109/JIOT.2019.2931647

Al-Kadi, O. S. (2015). A multiresolution clinical decision support system based on fractal model design for classification of histological brain tumours. Computerized Medical Imaging and Graphics,41, 67-79. https://doi.org/10.1016/j.compmedimag.2014.05.013

Angley, G. J., Nolan K. M., \& Nolan, T. W. (1992). The foundation of improvement. Silver Spring, MD: API Publishing.

Bardram, J. E., \& Bossen, C. (2005). A web of coordinative artifacts: collaborative work at a hospital ward. In: Proceedings of the 2005 international ACM SIGGROUP conference on Supporting group work. ACM, 168-176. https://doi.org/10.1145/1099203.1099235

Berwick, D. M. (1996). A primer on leading the improvement of systems. British Medical Journal, 312, 619-622. https://doi.org/10.1136/bmj.312.7031.619

Bornstein, B. H., \& Emler, A. C. (2001). Rationality in medical decision making: a review of the literature on doctors decision making biases. Journal of Evaluation in Clinical Practice, 7(2), 97-107. https://doi.org/10.1046/j.13652753.2001.00284.x

Campbell, D. A., Englesbe, M. J., Kubus, J. J., Phillips, L. R., Shanley, C. J., Velanovich, V., ... Share, D. A. (2010). Accelerating the pace of surgical quality improvement: the power of hospital collaboration. Archives of Surgery, 145(10), 985-991. https://doi.org/10.1001/archsurg.2010.220

Chanamool, N., \& Naenna, T. (2016). Fuzzy FMEA application to improve decision-making process in an emergency department. Applied Soft Computing, 43, 441-453. https://doi.org/10.1016/j.asoc.2016.01.007

Chang, I. C., Hwang, H. G., Hung, W. F., \& Li, Y. C. (2007). Physicians' acceptance of pharmacokinetics-based clinical decision support systems. Expert Systems with Applications, 33(2), 296-303. https://doi.org/10.1016/j.eswa.2006.05.001

Charles, C., Gafni, A., \& Whelan, T. (1997). Shared decision-making in the medical encounter: what does it mean? (Or it takes at least two to tango). Social Science \& Medicine, 44(5), 681-692. https://doi.org/10.1016/S02779536(96)00221-3

Chassin, M. (1998). Is health care ready for six sigma quality? Milbank Quarterly, 76(4), 565-591. https://doi.org/10.1111/1468-0009.00106

Conrad, D. A. (1993). Coordinating patient care services in regional health systems: the challenge of clinical integration. Hosp Health Serv Adm. 38(4), 491-508. http://www.ncbi.nlm.nih.gov/pubmed/10130609

Counte, M. A., \& Meurer, S. (2001). Issues in the assessment of continuous quality improvement implementation in health care organizations. International Journal for Quality in Health Care, 13(3), 197-201. https://doi.org/10.1093/intqhe/13.3.197

Cupryk, M. (2011). Standardizing patient safety risk management. Pharmaceutical Engineering, 11(2), 1-10.

Elwyn, G., Edwards, A., \& Kinnersley, P. (1999). Shared decision-making in primary care: the neglected second half of the consultation. British Journal of General Practice, 49, 477-482.

Elwyn, G., Edwards, A., Kinnersley, P., \& Grol, R. (2000). Shared decision making and the concept of equipoise: the competences of involving patients in healthcare choices. British Journal of General Practice, 50, 892-897.

Faraj, S., \& Xiao, Y. (2006). Coordination in fast-response organizations. Management Science, 52(8), 1155-1169. https://doi.org/10.1287/mnsc. 1060.0526

Ferlie, E. B., \& Shortell, S. M. (2001). Improving the quality of health care in the United Kingdom and the United States: a framework for change. Milbank Quarterly, 79(2), 281-315. https://doi.org/10.1111/1468-0009.00206

Ferrera, J. M. C., Cebada, E. C., \& Zamorano, L. R. M. (2014). The effect of quality and socio-demographic variables on efficiency measures in primary health care. The European Journal of Health Economics, 15(3), 289-302. https://doi.org/10.1007/s10198-013-0476-1

Gaynes, R., Richards, C., Edwards, J., Emori, T. G., Horan, T., Alonso-Echanove, J., ... Tolson, J. (2001). Feeding back 
surveillance data to prevent hospital-acquired infections. Emerging Infectious Diseases, 7(2), 295-298. https://doi.org/10.3201/eid0702.010230

Groothuis, S., Merode, G. V., \& Hasman, A. (2001). Simulation as decision tool for capacity planning. Computer Methods and Programs in Biomedicine, 66, 139-151. https://doi.org/10.1016/S0169-2607(00)00131-0

Grover, S., Agrawal, V. P., \& Khan, I. A. (2004). A digraph approach to TQM evaluation of an industry. International Journal of Production Research, 42(19), 4031-4053. https://doi.org/10.1080/00207540410001704032

Guinet, A., \& Chaabane, S. (2003). Operating theatre planning. Int. J. Production Economics, 85, 69-81. https://doi.org/10.1016/S0925-5273(03)00087-2

Gurumurthy, A., Mazumdar, P., \& Muthusubramanian, S. (2013). Graph theoretic approach for analysing the readiness of an organisation for adapting lean thinking a case study. International Journal of Organizational Analysis, 21(3), 396427. https://doi.org/10.1108/IJOA-04-2013-0652

Guyatt, Gordon, Armine Yalnizyan, and P. J. Devereaux (2002). Solving the public health care sustainability puzzle. Canadian Medical Association Journal, 167(1), 36-38.

Haraden, C., \& Resar, R. (2004). Patient flow in hospitals: understanding and controlling it better. Frontiers of Health Services Management, 20(4), 3-15. https://doi.org/10.1097/01974520-200404000-00002

Harper, P. R. (2002). A framework for operational modelling of hospital resources. Health Care Management Science, 5(3), 165-173. https://doi.org/10.1023/A:1019767900627

Herasevich, V., Pickering, B. W., Dong, Y., Peters, S. G., \& Gajic, O. (2010). Informatics infrastructure for syndrome surveillance, decision support, reporting, and modeling of critical illness. Mayo Clinic Proceedings, 85(3), 247-254. https://doi.org/10.4065/mcp.2009.0479

Hurtado, A. V., \& Greenlick, M. R. (1971). A disease classification system for analysis of medical care utilization, with a note on symptom classification. Health Service Research Fall, 6(3), 235-250.

Institute of Medicine Committee on the Future of Emergency Care in the US Health System. (2006). Hospital-based emergency care: at the breaking point. Washington, DC: The National Academic press.

Kilo, C. M. (1998). Framework for collaborative improvement: lessons from the institute for health care improvement's break through series. Quality Management in Health Care, 6(4), 1-13. https://doi.org/10.1097/00019514199806040-00001

Kim, K. H., Kim, K. S., Kim, D. Y., Kim, J. H., \& Kang, S. H. (2008). Brand equity in hospital marketing. Journal of Business Research, 61(1),75-82. https://doi.org/10.1016/j.jbusres.2006.05.010

Kollberg, B., Dahlgaard, J., \& Brehmer, P. (2007). Measuring lean initiatives in health care services: issues and findings. International Journal of Productivity and Performance Management, 56(1), 7-24. https://doi.org/10.1108/17410400710717064

Kuo, Y. F., \& James, S. G. (2011). Association of hospitalist care with medical utilization after discharge: evidence of cost shift from a cohort study. Annals of Internal Medicine 155(3), 152-159. https://doi.org/10.7326/0003-4819-155-3201108020-00005

Lee, W. I. (2010). The development of a qualitative dynamic attribute value model for healthcare institutes. Iranian Journal of Public Health, 39(4), 15-25.

Li, L., \& Benton, W. C. (2003). Hospital capacity management decisions: Emphasis on cost control and quality enhancement. European Journal of Operational Research, 146(3), 596-614. https://doi.org/10.1016/S03772217(02)00225-4

McGinnis, J. M., Stuckhardt, L., Saunders, R., \& Smith, M. (Eds.) (2013). Best care at lower cost: the path to continuously learning health care in America. National Academies Press.

McNeil, B. J., Pauker, S. G., Sox, H. C., \& Tversky, A. (1982). On the elicitation of preferences for alternative therapies. New England Journal of Medicine, 306, 1259-1262. https://doi.org/10.1056/NEJM198205273062103

Mitleton-Kelly, E. (2011). A complexity theory approach to sustainability: A longitudinal study in two London NHS hospitals. Learning Organization, 18(1), 45-53. https://doi.org/10.1108/09696471111095993

Moreno, L., Aguilar, R. M., Martın, C. A., Pineiro, J. D., Estevez, J. I., Sigut, J. F., Sanchez, J. L., ... Jimenez, V. I. (1999). Patient-centered simulation tool for aiding in hospital management. Simulation Practice and Theory, 7, 373393. https://doi.org/10.1016/S0928-4869(99)00007-5 
Nadkarni, V. M., Larkin, G. L., Peberdy, M. A., Carey, S. M., Kaye, W., Mancini, M. E., ... Berg, R. A. (2006). First documented rhythm and clinical outcome from in-hospital cardiac arrest among children and adults. The Journal of the American Medical Association, 295(1), 50-57. https://doi.org/10.1001/jama.295.1.50

Nazari, S., Fallah, M., Kazemipoor, H., \& Salehipour, A. (2018). A fuzzy inference-fuzzy analytic hierarchy processbased clinical decision support system for diagnosis of heart diseases. Expert Systems with Applications, 95, $261-$ 271. https://doi.org/10.1016/j.eswa.2017.11.001

Patel, V. L., Cytryn, K. N., Shortliffe, E. H., \& Safran, C. (2000). The collaborative health care team: the role of individual and group expertise. Teaching and Learning in Medicine: An International Journal, 12(3), 117-132. https://doi.org/10.1207/S15328015TLM1203_2

Piri, S., Delen, D., Liu, T., \& Zolbanin, H. M. (2017). A data analytics approach to building a clinical decision support system for diabetic retinopathy: Developing and deploying a model ensemble. Decision Support Systems, 101, 1227. https://doi.org/10.1016/j.dss.2017.05.012

Redelmeier, D. A., \& Eldar Shafir (1995). Medical decision making in situations that offer multiple alternatives. Journal of the American Medical Association, 273(4), 302-305. https://doi.org/10.1001/jama.1995.03520280048038

Safford, M. M., \& Allison, J. J. (2007). Patient complexity: more than comorbidity the vector model of complexity. Journal of General Internal Medicine, 382-390. https://doi.org/10.1007/s11606-007-0307-0

Sahinoglu, M., \& Wool, K. (2014). Risk assessment and management to estimate hospital credibility score of patient health care quality. Applied Cyber Physical System. https://doi.org/10.1007/978-1-4614-7336-7_13

Santelices, L. C., Wang, Y., Severyn, D., Druzdzel, M. J., Kormos, R. L., \& Antaki, J. F. (2010). Development of a hybrid decision support model for optimal ventricular assist device weaning. The Annals of Thoracic Surgery, 90(3), 713720. https://doi.org/10.1016/j.athoracsur.2010.03.073

Sloane, E. B., Liberatore, M. J., Nydick, R. L., Luo, W., \& Chung, Q. B. (2003). Using the analytic hierarchy process as a clinical engineering tool to facilitate an iterative, multidisciplinary, microeconomic health technology assessment. Computers \& Operations Research, 1447-1465. https://doi.org/10.1016/S0305-0548(02)00187-9

Stevenson, F. A., Barry, C. A, Britten, N., Barber, N., \& Bradley, C.P. (2000). Doctor-patient communication about drugs: the evidence for shared decision making. Social Science \& Medicine, 50, 829-840. https://doi.org/10.1016/S02779536(99)00376-7

Taheri, P. A., Butz, D. A., \& Greenfield, L. J. (2000). Length of stay has minimal impact on the cost of hospital admission. Journal of the American College of Surgeons, 191(2), 123-130. https://doi.org/10.1016/S10727515(00)00352-5

Thiéry, G., Azoulay, E., Darmon, M., Ciroldi, M., De Miranda, S., Lévy, V., ... Schlemmer, B. (2005). Outcome of cancer patients considered for intensive care unit admission: a hospital-wide prospective study. Journal of Clinical Oncology, 23(19), 4406-4413. https://doi.org/10.1200/JCO.2005.01.487

Trivedi, M. H., \& Daly, E. J. (2007). Measurement-based care for refractory depression: a clinical decision support model for clinical research and practice. Drug and Alcohol Dependence, 88, S61-S71. https://doi.org/10.1016/j.drugalcdep.2007.01.007

Veatch, R. M. (1972). Models for ethical medicine in a revolutionary age. The Hasting Center Report, 2(3), 5-7. https://doi.org/10.2307/3560825

Walczak, S., Pofahl, W. E., \& Scorpio, R. J. (2002). A decision support tool for allocating hospital bed resources and determining required acuity of care. Decision Support Systems, 34, 445-456. https://doi.org/10.1016/S01679236(02)00071-4

Wang, B. B., Wan, T. T., Clement, J., \& Begun, J. (2001). Managed care, vertical integration strategies and hospital performance. Health Care Management Science, 4(3),181-91. https://doi.org/10.1023/A:1011492731396

Wright, A., \& Sittig, D. F. (2008). A four-phase model of the evolution of clinical decision support architectures. International Journal of Medical Informatics, 77(10), 641-649. https://doi.org/10.1016/j.ijmedinf.2008.01.004

Wright, D. F., Anakin, M. G., \& Duffull, S. B. (2019). Clinical decision-making: An essential skill for 21st century pharmacy practice. Research in Social and Administrative Pharmacy, 15(5), 600-606. https://doi.org/10.1016/j.sapharm.2018.08.001

Xu, M., \& Shen, J. (2013). Clinical decision support model of heart disease diagnosis based on Bayesian networks and case-based reasoning. In The 19th International Conference on Industrial Engineering and Engineering 
Management (pp. 219-225). Springer, Berlin, Heidelberg. https://doi.org/10.1007/978-3-642-38391-5_23

Zhang, Y. F., Tian, Y., Zhou, T. S., Araki, K., \& Li, J. S. (2016). Integrating HL7 RIM and ontology for unified knowledge and data representation in clinical decision support systems. Computer Methods and Programs in Biomedicine, 123, 94-108. https://doi.org/10.1016/j.cmpb.2015.09.020

\section{Copyrights}

Copyright for this article is retained by the author(s), with first publication rights granted to the journal.

This is an open-access article distributed under the terms and conditions of the Creative Commons Attribution license which permits unrestricted use, distribution, and reproduction in any medium, provided the original work is properly cited. 